\title{
Telerobotic Surgery for Right and Sigmoid Colectomy
}

\author{
Arthur Rawlings, M.D., M.Div. and David Crawford, M.D. \\ University of Illinois College of Medicine at Peoria, Illinois
}

USA

\section{Introduction}

Surgery, unlike modern medicine, retains its link with ancient traditions. Human anatomy has not changed and the surgical approach to many diseases has remained the same for decades, even centuries. However, the techniques have evolved. For centuries, the sole approach to the disease was a large incision. Laparoscopic surgery ushered in a new era. Small incisions promised shorter hospital stays, less postoperative pain, better cosmesis, and a quicker return to normal activity. Patients now desire a minimally invasive surgical approach to their disease if at all possible.

The benefits to the patient were bought with the price of surgeons losing maneuverability in the operative field and having only a two-dimensional instead of a three-dimensional view. For many the transition was difficult. The learning curve was steep. In a short time, though, some procedures such as a laparoscopic cholecystectomy became the gold standard and basic laparoscopic skills were incorporated into general surgery training programs. The next major advance in minimally invasive surgery was the development of telemanipulation systems, also referred to as robots. Originally spearheaded in the United States by the Department of Defense, these robotic devices were intended for surgeons to operate from a remote location. These systems restored pitch and yaw at the end of the instruments, the two degrees of freedom lost with the use of traditional laparoscopic instruments. They also added other benefits such as tremor reduction, motion scaling, surgeon camera control, comfortable ergonomics, and a three-dimensional view of the operative field.

The first robotic system approved for intraabdominal surgery in the United States by the Food and Drug Administration (FDA) was the AESOP (Automated Endoscopic System for Optimal Position) system in 1993 (Oddsdottir \& Birgisson, 2004). AESOP (Computer Motion, Goleta, California) is a computerized robotic camera assistant for laparoscopic surgery. It has gone through several modifications since then and is still available today as a voice-activated, surgeon-controlled, camera assistant. It offers a stable camera platform but has no arm for direct manipulation or dissection of the tissues.

The first robotic system approved for intraabdominal surgery that did offer direct manipulation and dissection capabilities was the da Vinci system (Intitive Surgical, Inc., Sunnyvale, California). In 2000, the FDA approved the da Vinci system. Approval for a second system, ZEUS (Computer Motion, Goleta, California), quickly followed in 2001 
(Marescaux \& Rubino, 2004). Since then, the two companies have merged and ZEUS is no longer available. The da Vinci system is the only surgical platform available on the market that offers direct manipulation and dissection capabilities. Now, there are two generations of da Vinci robots in use: the da Vinci and the da Vinci S. These will be the systems focused on in this chapter.

\section{The da Vinci System}

The da Vinci system consists of three basic components: surgical cart, vision tower, and surgeon's console. The surgical cart is home to the three robotic arms. A fourth optional arm is also available. One arm is used for the camera, two arms are used for a variety of available surgical instruments, and the fourth optional arm is used mainly as an assistant for retraction. Laparoscopic surgery reduced the degrees of freedom in the abdomen to five: 1) in/out, 2) pitch (up/down), 3) yaw (left/right), 4) rotate, and 5) grasp. The robotic arms added 6) internal pitch and 7) internal yaw at the end of the instruments, restoring the full seven degrees of freedom available during an open abdominal case. The surgical cart to which the arms are attached is on rollers and can approach the operating table and be positioned over the patient from any direction necessary.

The surgeon's console consists of binocular monitors, foot pedals, and two hand-held masters used to manipulate the camera and surgical instruments. The binocular monitors can be switched during the case from a two-dimensional to a three-dimensional view with the press of a button. Having a three-dimensional view regains another component of open surgery that was lost with laparoscopic surgery. The surgeon sits at the console with his forearms and forehead resting on cushions, leaving his hands and feet free to operate the controls.

The vision tower contains the insufflator, light source, printer, camera, and computer hardware that generates the image. It also provides a stand for a monitor for the assistants in the room to watch during the case. The da Vinci S system also sports high definition vision in a panoramic 16:9 ratio along with an interactive video monitor called TilePro that allows a proctor to draw on the screen, thus giving visual direction to the person behind the surgeon's console.

\section{Robotic Colectomy}

The da Vinci system has been used for practically every intraabdominal procedure performed by general surgeons (Hanly \& Talamini, 2004; Ballantyne, 2007). Our focus is on the use of the da Vinci system for a colectomy.

\subsection{Our Background}

The Peoria Surgical Group is a university-affiliated, community training program with the University of Illinois College of Medicine at Peoria. There are three general surgery residents per year and no fellowships within the program. In 2002, the Peoria Surgical Group became the first private practice owner of the da Vinci system. At that time, the fourth arm was not available. It has since been purchased, but is presently not used for a right or sigmoid colectomy. As of August 2005, the chief of minimally invasive surgery, Dr. Crawford, had performed 109 cases with the da Vinci system. He has performed 34 
colectomies. The results of his first 30 colectomies have been published (Rawlings et al., 2006, 2007).

\subsection{Patient Selection}

Dr. Crawford's patients have the choice of three hospitals in Peoria, Illinois, two of which now have the da Vinci system. Robotic assistance is not discussed with the patients until they have chosen one of the robot's host hospitals. If a robot's host hospital is chosen, the patients are offered a choice between robotic and laparoscopic surgery if a minimally invasive approach is deemed appropriate. No patients are diverted to a robot's host hospital specifically for a robotic colectomy. Laparoscopic colectomies are performed at all three hospitals and on several occasions the second colectomy of the day at a robot's host hospital is performed laparoscopically instead of robotically because of staffing concerns expressed by the hospitals.

\subsection{Robotic Right Colectomy}

The patient is lying supine on a bean bag. The bag is positioned flush with the patient's right side, allowing excess bag on the left side with which to wrap the left side of the patient. The chest is secured circumferentially to the table with heavy tape at the level of the clavicles. The legs are secured at the thigh and calf with straps. Establishment of pneumoperitoneum, trocar placement (Figure 1), and initial exploration are performed in the supine position. The tattooed lesion or pathology is located and the planned point of transverse mesocolic division is marked based on the location of the right branch of the middle colic artery. The table is tilted to the left to allow the small intestine to fall off of the midline. The robot is then brought in over the right upper quadrant to dock with the camera port periumbilically and the right lower and left upper quadrant ports (See arrow, Figure 1). A five millimeter port is placed in the left lower quadrant and is used to grasp the ileocecal valve and to put the ileocolic vascular pedicle on tension. This pedicle is divided at the level of the duodenum with a vascular stapler that is brought in through the left-sided twelve millimeter port. The right mesocolon is mobilized off Gerota's fascia, the ureter is identified and then the ileal mesentery is divided with harmonic energy shears out to a point ten centimeters from the ileocecal valve. The mesocolic mobilization is then carried up to the duodenum and the transverse mesocolon. The transverse mesocolon is divided with harmonic shears, clips, and vascular staplers as needed. The transverse colon and ileum are then divided with a stapler from the left-sided twelve millimeter port. The colon remains attached to the right paracolic gutter to keep it from falling medially.

An isoperistaltic side-to-side anastomosis is then created between the ileum and transverse colon. A thirty centimeter long 2-0 silk suture on a Keith needle is used to join the distal transverse colon to the ileum six centimeters from the cut end. The Keith needle is then brought out through the abdominal wall in the right upper quadrant laterally and held with a hemostat. This keeps the anastomosis away from the camera and up in the air, making it easier to work on. The colon and ileum are also joined with suture near the cut end of the ileum. Harmonic shears are used to create an ileotomy and colotomy. Then a linear cutting stapler from the left-sided twelve millimeter port joins the bowel through these openings. The ileocolotomy is then sewn closed with running 2-0 Vicryl in two layers. The mesenteric defect is not closed. The suture holding the anastomosis up and to the right is cut, allowing the colon to fall medially out of the way. The right colon's lateral attachments are divided 
with cautery, and the specimen is extracted through the left-sided twelve millimeter port site after being converted to a small four centimeter muscle splitting incision (Solid line, Figure 1). This incision is protected from potential contamination with a short segment of a camera bag. Port sites are anesthetized and closed in the usual manner.

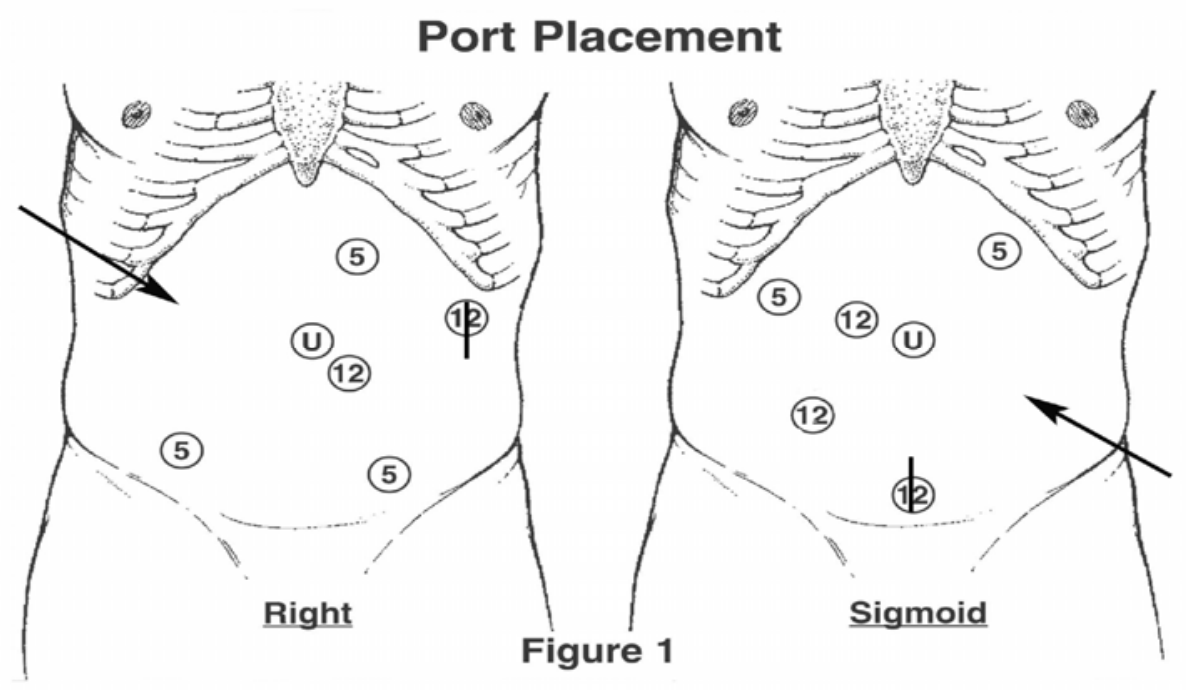

Figure 1. Port Placement for a Robotic Colectomy. The arrows show the angle of approach by the surgical cart. The number in the circle indicates to size of trocar. The " $U$ " is the umbilicus. The solid line through the $12 \mathrm{~mm}$ trocar sites is the specimen extraction site

\subsection{Robotic Sigmoid Colectomy}

The patient is in supine lithotomy position with the anterior thighs in the same plane as the abdominal wall. The bean bag is embracing the patient's right side. The chest is secured circumferentially to the table with heavy tape at the level of the clavicles. Establishment of pneumoperitoneum, trocar placement (Figure 1), and initial exploration are performed in the supine position. The patient is tilted steeply to the right and the tattooed lesion or pathology is localized. Reverse Trendelenburg position is added to the right tilt and the surgical robot is brought up to the table in the left lower quadrant (See arrow, Figure 1). The robot's left arm goes in the left upper quadrant five millimeter port. The camera arm is in the periumbilical port. A five millimeter port remains on the robot's right arm and is inserted through the twelve millimeter suprapubic port. The splenic flexure is completely mobilized. All three arms are then adjusted as the patient is put into Trendelenburg position, along with the right arm and its five millimeter port being moved to the right lower quadrant twelve millimeter port. The inferior mesenteric artery is identified and isolated from a medial to lateral approach, and the left ureter is identified. All mobilization of the colon and mesorectal division is completed using electrocautery and harmonic energy. The robot is disengaged and endoscopic staplers are used to divide the inferior mesenteric artery and rectum. The specimen is externalized through the extended suprapubic port site that is protected from potential contamination with a short segment of 
a camera bag (Solid line, Figure 1). The specimen is resected, the stapler anvil introduced, and the proximal colon is dropped back in the abdomen. The fascia is closed and pneumoperitoneum reestablished. A standard end-to-end anastomosis is performed using conventional laparoscopy, and tested in the usual manner. Port sites are anesthetized and closed in the usual manner.

Port placement in males and females is identical. Initially, when only eight millimeter instruments were available for the da Vinci system, the ports were placed through twelve millimeter dilating trocars. This allowed the assistants at the table to advance or retract the robot trocars/arms to optimize instrument use with less fear of dislodging the robot trocar from the abdominal wall. The switch to five millimeter instruments took place November of 2004, when these instruments became available to us. The five millimeter instruments handle differently, but were easy to adjust to. Five millimeter instruments are now routinely used in order to minimize incision size. The only place that the trocar through a trocar technique is still used is in a sigmoid colectomy between the suprapubic and right lower quadrant sites. The scrub staff makes this exchange without the surgeon returning to the field.

\subsection{Post Operative Care}

The patients are placed on clear liquids the day of surgery and receive a single dose of Milk of Magnesia on the morning of postoperative day one. All robotic colectomies are placed on a hospital-wide activity protocol. All patients have a patient controlled analgesia pump for pain control that is discontinued when they tolerate liquids well. Patients are discharged when tolerating liquids well, voiding, moving their bowels, and having adequate pain control with oral analgesics.

\section{Review of Literature}

As stated earlier, the da Vinci system has been used for practically every intraabdominal procedure performed by general surgeons (Hanly \& Talamini, 2004; Ballantyne, 2007). We will limit our discussion to colectomy.

\subsection{Robotic Colectomy}

Webber reported performing the first robotic colon resection in 2001 using the da Vinci system (Webber et al. 2002). Since then, at least fifteen other studies of robotic colectomy have been reported. The published series are listed in table 1 . The difficulty in comparing one series to another is that a wide range of procedures are included in some series and the parameters reported vary from one study to another. For example, Giulianotti included abdominoperineal resections and ileocecal resections along with right and left hemicolectomies while Woeste reported only sigmoid colectomies (Giulianotti et al., 2003; Woeste et al., 2005). Delany reported times from anesthesia to incision and incision to extubation whereas we reported times from initial incision to robot engagement, from robot engagement to robot disengagement, and from robot disengagement to the end of the case. (Delany et al., 2003; Rawlings et al., 2006, 2007).

Among all of these studies are four that specifically compare a laparoscopic colectomy with a robotic colectomy (Anvari et al., 2004; D'Annibale et al., 2003; Delany et al., 2003; Rawlings et al., 2007). Since a laparoscopic colectomy is the only competition a robotic colectomy has if the patient wants a minimally invasive approach, we will focus on these four studies. 


\begin{tabular}{|l|c|c|l|c|}
\hline First Author & YEAR & Number & Operative Time* & Conversions \\
\hline Weber & 2002 & 2 & $284(228-340)$ & 0 \\
\hline Hashizume & 2002 & 3 & $260(180-335)$ & 0 \\
\hline Delaney & 2003 & 6 & $217(170-274)$ & 1 \\
\hline Giulianotti & 2003 & 16 & $211(90-360)$ & 0 \\
\hline Vibert & 2003 & 3 & $380(330-450)$ & 0 \\
\hline Ewing & 2004 & 12 & $248(180-350)$ & 0 \\
\hline Hanly & 2004 & 35 & $177(\mathrm{NA})$ & 5 \\
\hline Ayav & 2004 & 5 & $265(180-240)$ & 0 \\
\hline Anvari $\ddagger$ & 2004 & 10 & $155 \pm 14$ & 0 \\
\hline D'Annibale & 2004 & 53 & $240 \pm 61$ & 5 \\
\hline Hubens & 2004 & 7 & NA & 0 \\
\hline Woeste & 2004 & 4 & $237 \pm 6$ & 2 \\
\hline Braumann & 2005 & 5 & $201(80-300)$ & 0 \\
\hline Ayav & 2005 & 6 & $172(45-280)$ & 1 \\
\hline Anvari & 2005 & 6 & $109(90-160)$ & 2 \\
\hline Rawlings & $2006 \& 2007$ & 30 & $226(90-340)$ & 1 \\
\hline
\end{tabular}

* Average (Range) or \pm Standard Deviation in Minutes, NA: Not Available

$\ddagger$ The only study using the ZEUS system

Table 1. Robotic Colectomy Series Articles in Chronological Order of Publication

\subsection{Robotic verses Laparoscopic Colectomy}

Anvari used the ZEUS system to compare ten robotic and ten consecutive laparoscopic colectomies (Anvari et al., 2004). Although the ZEUS system is no longer available, the authors reported one definite advantage of the system for a colectomy that highlights one of the deficiencies of the da Vinci system. The ZEUS system integrated the robot and the operating room table so the patient could be tilted or rotated without any readjustment of the robotic arms. The da Vinci is not integrated with the operating room table. Rotating or tilting the patient is not impossible with the da Vinci system. It is just very inconvenient.

D'Annibale has the largest comparison to date of robotic and laparoscopic colectomies, with fifty-three consecutive robotic patients and fifty-three matched laparoscopic patients (D'Annibale et al., 2003). The authors reported no significant difference in operative time with $240 \pm 61$ minutes for robotic cases and $222 \pm 77$ minutes for laparoscopic cases. There was, however, a statistically significant difference in system and patient setup time. It took $24 \pm 12$ minutes to set up for a robotic case but only $18 \pm 7$ minutes for a laparoscopic case $(p=0.002)$. Their length of hospital stay for robotic cases averaged ten days and did not differ from a matched laparoscopic group. Though favorable toward the da Vinci system over traditional laparoscopy for specific stages of the procedure, they concluded their study stating that, "investigations are needed to evaluate the cost-effectiveness of this approach." The third comparison study does include cost. In 2003, Delany published a comparison of six robotic operations with procedure matched control laparoscopic patients (Delany et al., 2003). Total hospital cost was higher for the robotic group, but this did not reach statistical 
significance. They attributed this to their small sample size. They stated that the operating room and equipment cost was approximately $\$ 350$ higher for the robotic group, to cover the robotic instruments and sterile drapes. Their study did include disposable operating cost, but did not include the acquisition or maintenance costs of the da Vinci system, nor was cost a major focus of their publication.

In our comparison, the fourth published, we had a significant focus on cost, breaking it down into total hospital cost, operating room cost, operating room supply cost, and operating room personnel cost (Rawlings et al., 2007). All of our costs were adjusted to 2005 US dollars. We showed that each cost category was higher for the robotic group over the laparoscopic group (Tables 2 \& 3). Despite our sample size being twice as large as Delany's sample size for right and sigmoid colectomy, a statistically significant increase in our study was lacking in several cost categories. For a right colectomy, there was a significant difference in all the operating room cost categories, but this did not result in a significant difference for total hospital cost. For a sigmoid colectomy, a significant difference was only reached in operating room personnel and supply costs. The remaining cost categories for a sigmoid colectomy did not reach statistical significance. This is undoubtedly due to our small sample size. To illustrate, the analysis of our total hospital cost had a power of only $6 \%$ for a sigmoid colectomy and $12 \%$ for a right colectomy in showing a statistically significant difference. Using our existing data set and assuming a similar ratio of robotic and laparoscopic cases, there would have to be a total of 1,616 sigmoid colectomy cases and 391 right colectomy cases to reach a desired power of $80 \%$ with a significant difference of $p<0.05$ in total hospital cost. Needless to say, with these numbers, a randomized controlled trial comparing the cost of a laparoscopic with a robotic colectomy is well beyond the ability of any one institution in the near future.

Even though we had a significant focus on cost in our study, we did not include the acquisition and maintenance costs of the da Vinci system. In our setting, these costs cannot be directly passed on to the patient. We can, however, charge the patient for the robot disposables such as drapes and the robotic instruments that have a ten to twenty case lifetime. At our institution we are presently required to have two circulating nurses in all robotic cases instead of one, as we normally have in a laparoscopic case. This helps explain the difference in operating room personnel cost between robotic and laparoscopic cases. As far as the acquisition and maintenance costs go, they are presently viewed as capital expenses. They are not directly passed on to the patient undergoing a robotic procedure, in the same way we do not directly pass on the cost of the handicapped access ramp only to the patients who are wheelchair bound. To our knowledge, no one has studied and published the true cost-to-benefit ratio of the da Vinci system. This is a very complex economic issue, but could be a very fruitful area of investigation.

\begin{tabular}{|l|c|c|c|}
\hline \multicolumn{4}{|c|}{ RIGHT COLECTOMY - COST ANALYSIS* } \\
\hline & Laparoscopic $(\mathbf{n}=\mathbf{1 5})$ & Robotic $(\mathbf{n}=\mathbf{1 7})$ & $\boldsymbol{p}$ value \\
\hline Total Hospital Cost & $\$ 8,073 \pm 2,805$ & $\$ 9,255 \pm 5,075$ & 0.430 \\
\hline Total OR Cost & $\$ 4,339 \pm 867$ & $\$ 5,823 \pm 907$ & $<0.000$ \\
\hline OR Personnel Cost & $\$ 1,340 \pm 402$ & $\$ 2,048 \pm 309$ & $<0.000$ \\
\hline OR Supply Cost & $\$ 1,841 \pm 518$ & $\$ 2,950 \pm 475$ & $<0.000$ \\
\hline OR Time Cost & $\$ 990 \pm 300$ & $\$ 1,521 \pm 321$ & $<0.000$ \\
\hline
\end{tabular}

Table 2. Cost Analysis for a Right Colectomy: *Adjusted to 2005 US Dollars 


\begin{tabular}{|l|c|c|c|}
\hline \multicolumn{4}{|c|}{ SIGMOID COLECTOMY - COST ANALYSIS* } \\
\hline & Laparoscopic $(\mathbf{n}=\mathbf{1 2})$ & Robotic $(\mathbf{n}=\mathbf{1 3})$ & $\boldsymbol{p}$ value \\
\hline Total Hospital Cost & $\$ 10,697 \pm 11,719$ & $\$ 12,335 \pm 12,162$ & 0.735 \\
\hline Total OR Cost & $\$ 4,974 \pm 1,596$ & $\$ 6,059 \pm 1,225$ & 0.068 \\
\hline OR Personnel Cost & $\$ 1,621 \pm 617$ & $\$ 2,134 \pm 432$ & 0.024 \\
\hline OR Supply Cost & $\$ 2,137 \pm 905$ & $\$ 3,159 \pm 637$ & 0.003 \\
\hline OR Time Cost & $\$ 1,348 \pm 681$ & $\$ 1,500 \pm 461$ & 0.519 \\
\hline
\end{tabular}

Table 3. Cost Analysis for a Sigmoid Colectomy: *Adjusted to 2005 US Dollars

In our study, the robotic and laparoscopic groups for a right and sigmoid colectomy were similar in gender, age, body mass index, and indications for surgery. The average case time for a robotic right colectomy was 219 minutes, which was 50 minutes longer than the 169 minute average for the laparoscopic group. This was statistically significantly longer $(p=0.002)$. Two factors contributed to a longer average case time in the robotic cases. Our first study showed that the robotic port setup time - the time from initial insufflation with a Veress needle until the surgeon sits at the robot's console - for a right colectomy averaged 30 minutes (Rawlings et al., 2006). Also, an isoperistaltic side-to-side anastomosis was performed intracorporeally with robotic assistance, whereas an extracorporeal bowel resection and anastomosis was performed in the laparoscopic cases. The port setup time and the variation in anastomotic technique accounts for the robotic cases requiring more time than the laparoscopic cases in a right colectomy. In contrast, the average case time for a robotic sigmoid colectomy was 225 minutes, which was 26 minutes longer than the 199 minute average for the laparoscopic group. This was not statistically significant $(p=0.128)$. The robotic and laparoscopic sigmoid colectomy cases were performed in similar sequence, and the additional time for the robotic cases is mainly attributed to the 30 minute port setup time for the robot (Rawlings et al., 2006).

Our length of stay did not differ between the two comparison groups. A laparoscopic right colectomy averaged 5.5 days and a robotic right colectomy averaged 5.2 days $(p=0.862)$. A laparoscopic sigmoid colectomy averaged 6.6 days and a robotic sigmoid colectomy averaged 6.0 days $(\mathrm{p}=0.854)$. We also showed no difference in estimated blood loss between our comparison groups.

\section{Reflections on the da Vinci for Colectomy}

At least fifteen published series have demonstrated the technical feasibility of using the da Vinci system for performing a colon resection (Table 1). The use of this system for this procedure has definite advantages and disadvantages.

\subsection{Colectomy: The da Vinci Advantages}

There are several advantages of using the da Vinci system. The first is the enhanced visualization of the operative field. The da Vinci system allows the surgeon to choose between two-dimensional and three-dimensional representations of the operative field with its stereoscopic camera. The three-dimensional view is particularly helpful in depth of field and clarity of tissue planes during dissection. The camera also allows for a ten-fold magnification compared to the two-fold on standard laparoscopic cameras. 
The second advantage is that the surgeon has control over the camera by toggling a switch with a foot pedal. This allows the surgeon to place the camera at the location he desires when he desires it, rather than depending on an assistant to anticipate his desired view or respond to a command.

The third advantage is the wristed instruments, which introduce two more degrees of freedom into the operative field. Traditional laparoscopic surgery reduced the maneuverability in the operative field to only five degrees: 1) in-out, 2) rotation of shaft, 3) pitch (up-down), 4) yaw (left-right), and 5) grasp. The robotic instruments add two more degrees of freedom: 1) internal pitch at the end of the wristed instruments and 2) internal yaw at the end of the wristed instruments. In essence, the robot restores the maneuverability available during an open case with the surgeon's hand in the operative field to a laparoscopic case. It is like having your hands back in the field. It should also be mentioned that the robotic software provides tremor reduction and motion scaling to the wristed instruments, both significant advantages in delicate dissection and fine suturing situations.

Another advantage is the reduction of the surgeon's fatigue. During the robotic portion of the case, the surgeon is sitting with her forearms resting comfortably on a pad and her head resting against the console. Fatigue is also reduced because the hand controls can be recentered while leaving the surgical instruments in their present location. In traditional laparoscopic surgery, the surgeon had to move her hands to whatever location is needed to position the surgical instruments at their proper locations. This, at times, requires some rather awkward movements and pushes the limits of one's flexibility and reach. With the recentering feature of the da Vinci system, the surgeon can relocate her hands back to a normal position and then resume working. It is like lifting up the computer mouse and putting it back to a comfortable spot while leaving the cursor at its present location on the computer screen.

Finally, it should be mentioned that using the da Vinci system in the present surgical climate in the United States does allow one to be promoted as a "regional Minimally Invasive Surgery (MIS) expert," which is often a unique marketing opportunity. This is another aspect of the total cost-to-benefit ratio that comes from an institution or surgical group owning the da Vinci system. The perception is that having the da Vinci system enlarges your referral base, but this would be very hard to demonstrate.

\subsection{Colectomy: The da Vinci Disadvantages}

There are several general disadvantages of using the da Vinci system that apply to its use for any case. These include loss of tactile sensation, difficult team communication with the surgeon sequestered behind the console away from the patient and staff, increased room size requirements to accommodate the equipment, and cost of the device.

There are three distinct disadvantages of using the da Vinci system for a colectomy. The first is the inconvenience of altering port placement of the camera and instruments during the case. In the traditional laparoscopic approach, one easily pulled the camera out and placed it in another port that would accommodate it if another perspective were desired. Changing which port the camera or instruments enter the patient is not impossible with the da Vinci system. It is just very inconvenient. This precludes taking a quick look around from another port site during the robotic portion of the procedure. It also keeps one from quickly shifting an instrument from one port site to another to gain a different angle of attack for dissection. 
A second disadvantage is the difficulty encountered with working in the far lateral extensions of the operative field. For example, while working in the left lateral most aspect of the field (splenic flexure) the instrument in the right port (suprapubic) may reach its inner most limits before reaching the desired spot, and visa versa. Working with an instrument tip too close to the end of the port can also limit the ability of the instrument to function optimally. In traditional laparoscopic surgery, this is sometimes overcome by switching the camera and operating port for a portion of the case. That would be very inconvenient with the da Vinci system. Knowing this places a greater burden on proper port placement for the robotic case because the location determines how far laterally one may reasonably work in the surgical field. The port setup is the key aspect of any smoothly running robotic procedure. There are definite limits in movement with the robotic arm compared to the standard laparoscopic approach. For example, the robotic arm has a definite extension end point, whereas a few centimeters can be gained in traditional laparoscopic surgery by depressing the insufflated abdomen.

A third disadvantage in using the da Vinci system with this particular type of case is the inconvenience of rotating or tilting the patient. In minimally invasive procedures, the patients are sometimes tilted or rotated to allow gravity to help pull the organs that obstruct the operative field out of the way. Every change in the patient's position requires each arm of the robot to be reset to a new location. So, other forms of retraction must be implemented to compensate. Usually this entails another port for retraction. This assistant port can also be used to introduce objects into the field such as sutures, staplers, and measuring tapes. It is also used to remove the pathological specimen. This assistant port is usually not placed solely for retraction, but it will be used for retraction and the port will require surgical personnel to staff it.

\section{Conclusion}

The first reported robotic colectomy was performed in March, 2001. Since then, fourteen other studies have been published showing the safety and feasibility of using the da Vinci system for a colectomy and one study showed the safety and feasibility of using the ZEUS system. No robot specific complication has yet to be reported. There are definite advantages as well as disadvantages of using the da Vinci system for a colectomy. Though studies have been done comparing the cost of a laparoscopic colectomy with a robotic colectomy, more work needs to be done looking at cost from an even wider perspective than just cost per case. Is there any true marketing advantage to an institution having the da Vinci system? How does the cost of the robot as well as the cost of ongoing company support figure into the picture? The da Vinci system has been used for colectomy for less than a decade. We are optimistic that refinements in this system will make it even more attractive in the future for a right or sigmoid colectomy.

\section{Acknowledgements}

The surgical technique descriptions, figures, and the advantages and disadvantages discussion are used with kind permission from Springer Science and Business Media, Springer New York, Surgical Endoscopy, Vol. 20, No. 11, 2007, pp. 1713-1718, Telerobotic Surgery for Right and Sigmoid Colectomies: 30 Consecutive Cases, Rawlings, A.; Woodland, J. \& 
Crawford, D. and Surgical Endoscopy, Vol. 21, No. 10, 2007, pp. 1701-1708, Robotic versus Laparoscopic Colectomy, 2007, Rawlings, A.; Woodland, J.; Vegunta, R. \& Crawford, D.

\section{References}

Anvari, M.; Birch, D.; Bamehriz, F.; Gryfe, R. \& Chapman T. (2004). Robotic-assisted Laparoscopic Colorectal Surgery, Surgical Laparoscopy, Endoscopy E Percutaneous Techniques, Vol. 14, No. 6, (December 2004), pp. 311-315, ISSN 1530-4515

Anvari, M.; McKinley, C. \& Stein, H. (2005). Establishment of the World's First Telerobotic Remote Surgical Service for Provision of Advanced Laparoscopic Surgery in a Rural Community. Annals of Surgery, Vol. 241, No. 3, (March 2005), pp. 460-464, ISSN 0003-4932

Ayav, A.; Bresler, L.; Brunaud, L. \& Boissel, P. (2004). Early Results of One-Year Robotic Surgery Using the Da Vinci System to Perform Advanced Laparosopic Procedures. Journal of Gastrointestinal Surgery, Vol. 8, No. 6, (September 2004), pp. 720-726, ISSN 1091-255X

Ayav, A.; Bresler, L.; Hubert, J.; Brunaud, L. \& Boissel, P. (2005). Robotic-Assisted Pelvic Organ Prolapse Surgery. Surgical Endoscopy, Vol. 19, No. 9, (September 2005), pp. 1200-1203, ISSN 0930-2794

Ballantyne, G. (2007). Telerobotic Gastrointestinal Surgery: Phase 2 - Safety and Efficacy. Surgical Endoscopy, Vol. 21, No. 7, (July 2007), pp. 1054-1062, ISSN 0930-2794

Braumann, C.; Jacobi, C.; Menenakos, C.; Borchert, U.; Rueckert, J. \& Mueller, J. (2005). Computer-Assisted Laparoscopic Colon Resection with the Da Vinci System: Our First Experiences. Diseases of the Colon and Rectum, Vol. 48, No. 9, (September 2005), pp. 1820-1827, ISSN 0012-3706

D’Annibale, A.; Morpurgo, E.; Fiscon, V.; Trevisan, P.; Sovernigo, G.; Orsini, C. \& Guidolin, D. (2004). Robotic and Laparoscopic Surgery for Treatment of Colorectal Diseases. Diseases of the Colon and Rectum, Vol. 47, No. 12, (December 2004), pp. 2162-2168, ISSN 0012-3706

Delaney, C.; Lynch, A.; Senagore, A. \& Fazio, V. (2003). Comparison of Robotically Performed and Traditional Laparoscopic Colorectal Surgery. Diseases of the Colon and Rectum, Vol. 46, No. 12, (December 2003), pp. 1633-1639, ISSN 0012-3706

Giulianotti, P.; Coratti, A.; Angelini, M.; Sbrana, F.; Cecconi, S.; Balestracci, T. \& Caravaglois, G. (2003). Robotics in General Surgery: Personal Experience in a Large Community Hospital. Archives of Surgery, Vol. 138, No. 7, (July 2003), pp. 777-784, ISSN 00040010

Hanly, E. \& Talamini, M. (2004). Robotic Abdominal Surgery. The American Journal of Surgery, Vol. 188, No. 4A, (Supplemental to October 2004), pp. 19S-26S, ISSN 00029610

Hashizume, M.; Shimada, M.; Tomikawa, M.; Ikeda, Y.; Takahashi, I.; Abe, R.; Foga, F.; Gotoh, N.; Konishi, K.; Maehara, S. \& Sugimachi, K. (2002). Early Experiences of Endocsopic Procedures in General Surery Assisted by a Computer-enhanced Surgical System. Surgical Endoscopy, Vol. 16, No. 8, (August 2002), pp. 1187-1191, ISSN 0930-2794

Hubens, G.; Ruppert, M.; Balliu, L. \& Vaneerdeweg, W. (2004). What Have We Learnt After Two Years Working with the Da Vinci Robot System in Digestive Surgery? Acta chirurgica Belgica, Vol. 104, No. 6, (November 2004), pp. 609-614, ISSN 0001-5458 
Marescaux, J. \& Rubino, F. (2004). The ZEUS Telerobotic Surgical System, In: Primer of Robotic \& Telerobotic Surgery, Ballantyne, G.; Marescaux, J. \& Giulianotti, P. (Ed.), pp. 61-65, Lippincott Williams \& Wilkins, ISBN 0-7817-4844-5, Philadelphia

Oddsdottir, M. \& Birgisson, G. (2004). AESOP: A Voice-Controlled Camera Holder, In: Primer of Robotic \& Telerobotic Surgery, Ballantyne, G.; Marescaux, J. \& Giulianotti, P. (Ed.), pp. 35-41, Lippincott Williams \& Wilkins, ISBN 0-7817-4844-5, Philadelphia

Rawlings, A.; Woodland, J. \& Crawford, D. (2006). Telerobotic Surgery for Right and Sigmoid Colectomies: 30 Consecutive Cases. Surgical Endoscopy, Vol. 20, No. 11, (2006), pp. 1713-1718, ISSN 0930-2794

Rawlings, A.; Woodland, J.; Vegunta, R. \& Crawford, D. (2007). Robotic versus Laparoscopic Colectomy. Surgical Endoscopy, Vol. 21, No. 10, (2007), pp. 1701-1708, ISSN 09302794

Vibert, E.; Denet, C. \& Gayet, B. (2003). Major Digestive Surgery Using a Remote-Controlled Robot: The Next Revolution. Archives of Surgery, Vol. 138, No. 9, (September 2003), pp. 1002-1006, ISSN 0004-0010

Weber, P.; Merola, S.; Wasielewski, A. \& Ballantyne, G. (2002). Telerobotic-Assisted Laparoscopic Right and Sigmoid Colectomies for Benign Disease. Diseases of the Colon and Rectum, Vol. 45, No. 12, (December 2002), pp. 1689-1696, ISSN 0012-3706

Woeste, G.; Bechstein, W. \& Wullstein, C. (2005). Does Telerobotic Assistance Improve Laparoscopic Colorectal Surgery? International Journal of Colorectal Disease, Vol. 20, No.3, (May 2005), pp. 253-257, ISSN 0179-1958 


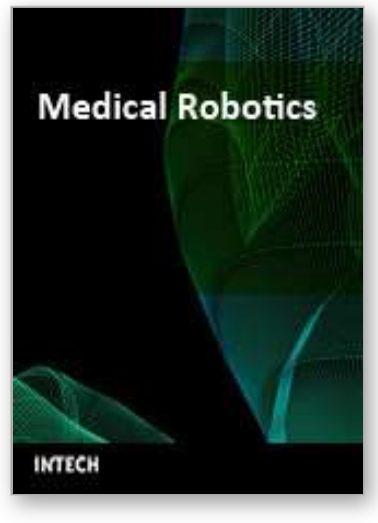

\author{
Medical Robotics \\ Edited by Vanja Bozovic
}

ISBN 978-3-902613-18-9

Hard cover, 526 pages

Publisher I-Tech Education and Publishing

Published online 01, January, 2008

Published in print edition January, 2008

The first generation of surgical robots are already being installed in a number of operating rooms around the world. Robotics is being introduced to medicine because it allows for unprecedented control and precision of surgical instruments in minimally invasive procedures. So far, robots have been used to position an endoscope, perform gallbladder surgery and correct gastroesophogeal reflux and heartburn. The ultimate goal of the robotic surgery field is to design a robot that can be used to perform closed-chest, beating-heart surgery. The use of robotics in surgery will expand over the next decades without any doubt. Minimally Invasive Surgery (MIS) is a revolutionary approach in surgery. In MIS, the operation is performed with instruments and viewing equipment inserted into the body through small incisions created by the surgeon, in contrast to open surgery with large incisions. This minimizes surgical trauma and damage to healthy tissue, resulting in shorter patient recovery time. The aim of this book is to provide an overview of the state-of-art, to present new ideas, original results and practical experiences in this expanding area. Nevertheless, many chapters in the book concern advanced research on this growing area. The book provides critical analysis of clinical trials, assessment of the benefits and risks of the application of these technologies. This book is certainly a small sample of the research activity on Medical Robotics going on around the globe as you read it, but it surely covers a good deal of what has been done in the field recently, and as such it works as a valuable source for researchers interested in the involved subjects, whether they are currently "medical roboticists" or not.

\title{
How to reference
}

In order to correctly reference this scholarly work, feel free to copy and paste the following:

Arthur Rawlings and David Crawford (2008). Telerobotic Surgery for Right and Sigmoid Colectomy, Medical Robotics, Vanja Bozovic (Ed.), ISBN: 978-3-902613-18-9, InTech, Available from:

http://www.intechopen.com/books/medical_robotics/telerobotic_surgery_for_right_and_sigmoid_colectomy

\section{INTECH}

open science | open minds

\section{InTech Europe}

University Campus STeP Ri

Slavka Krautzeka 83/A

51000 Rijeka, Croatia

Phone: +385 (51) 770447

\section{InTech China}

Unit 405, Office Block, Hotel Equatorial Shanghai

No.65, Yan An Road (West), Shanghai, 200040, China

中国上海市延安西路65号上海国际贵都大饭店办公楼 405 单元

Phone: +86-21-62489820 
Fax: +385 (51) 686166

Fax: +86-21-62489821

www.intechopen.com 
(C) 2008 The Author(s). Licensee IntechOpen. This chapter is distributed under the terms of the Creative Commons Attribution-NonCommercialShareAlike-3.0 License, which permits use, distribution and reproduction for non-commercial purposes, provided the original is properly cited and derivative works building on this content are distributed under the same license. 